\title{
Parametrization of Joint OFDM-based Radar and Communication Systems for Vehicular Applications
}

\author{
Martin Braun*, Christian Sturm ${ }^{\ddagger}$, Andreas Niethammer*, Friedrich K. Jondral* \\ ${ }^{*}$ Communications Engineering Lab, Karlsruhe Institute of Technology (KIT), Germany \\ ${ }^{\ddagger}$ Institut für Hochfrequenztechnik und Elektronik, Karlsruhe Institute of Technology (KIT), Germany \\ \{martin.braun, andreas.niethammer, friedrich.jondral\}@kit.edu, christian.sturm@ihe.uka.de
}

\begin{abstract}
The design process of a joint radar and communication system employing OFDM is analyzed. In particular, the question on how to choose OFDM modulation parameters such as carrier distance, guard interval length, frame length or pilot design is studied. Such systems use the same OFDM frames to transmit data and image the surroundings, so both radar and communication are affected by the choice of these parameters. The main design criteria for the radar component are accuracy of distance and speed measurements, whereas the communication subsystem needs to be designed such that it transmits robustly through a mobile communication channel. As these design criteria often are antipodal, arguments for choosing a particular set of modulation parameters are discussed. The presented design process is applied to the case of a vehicular (car-to-car) communication, in which moving participants communicate in the $24 \mathrm{GHz}$ range.
\end{abstract}

\section{INTRODUCTION}

Over the last few years, automotive safety systems have more and more become an intensive domain of research for RF engineers. Vehicles are equipped with mobile communication systems in order to share information about the traffic situation, current events concerning the traffic flow and possibly even less critical data such as information and entertainment services. Development of these car-to-car communication systems has made some progress recently, and the allocation of spectrum dedicated to automotive communication in the US and Europe has made an appearance of communicating cars on the worldwide markets quite likely. The dominating standard for inter-vehicular communication is IEEE 802.11p, operating in the frequency range around $5.9 \mathrm{GHz} .802 .11 \mathrm{p}$ is very closely related to the 802.11 a standard.

The other major area of development are vehicular radar systems. Many driver assistance systems such as adaptive cruise control (ACC), lane change assistance (LCA) or blind spot detection use radar to detect and track other targets in the near environment.

From a purely physical point of view, these systems are nearly identical: in both cases, transmitters and receivers of electromagnetic (EM) waves are used to emit or receive information. Using one system to do both radar and communication is therefore an obvious idea, and has in fact been proposed repeatedly in the past, even based on OFDM waveforms (e.g. [1]). A joint vehicular communication and radar system in the $24 \mathrm{GHz}$ band has also been described in [2], which uses FMCW and spread spectrum techniques instead of OFDM.

A recent approach towards fusing these two systems has been OFDM radar as proposed in [3]. OFDM radar uses a transmission scheme which emits bursts of data (subsequently called frames) when transmitting information and detects reflections of the own signal at the same time. Relative velocity and distance of the reflecting targets cause a modification of the received signal, which can be used to determine a radar image. Since the OFDM frames must be suitable for both radar image evaluation and robust data transmission, special attention must be paid to the parametrization of the OFDM modulation and frame design.

For the scope of this paper, all parameters shall be considered freely changeable, not having to follow any a-priori constraints. This differs from the design of $802.11 \mathrm{p}$, where one design goal was to maximise resemblance to 802.11a.

This paper is organized as follows: in Sections II, III and IV the constraining factors of the propagation channel, the radio system and the radar shall be explained and defined. Section $\mathrm{V}$ gives an example of how such a system can be designed, and an exemplary setup is given together with simulative verifications. Section VI concludes this paper.

\section{Mobile MUlTiPATH CHANNEL CHARACTERISTICS}

The propagation channel, which imposes most of the design criteria, is physically given and thus shall be analyzed first. Throughout this paper, the following channel model shall be used:

$$
r(t)=\sum_{l=0}^{L-1} c_{l} s\left(t-\tau_{l}\right) e^{j 2 \pi f_{D, l}}+n(t)
$$

$r(t)$ is the received signal, $s(t)$ the signal emitted from the transmitter, $L$ the number of propagation paths. $\tau_{l}, c_{l}$ and $f_{D, l}$ are the path delay, the complex attenuation and the Doppler shift of the $l$-th path, respectively. $n(t)$ is a noise signal.

Analyzing such channels is always a difficult task, since an exact mathematical representation is, in general, not known. The most accurate way to gather information about mobile channels in the $24 \mathrm{GHz}$ range would be to perform actual measurements between vehicles in different traffic situations; 
however, at this early stage of research, a more theoretical analysis is more appropriate. To bridge the gap, two sources of information were employed: ray tracing simulations of traffic situations, in which wave propagation paths are explicitly calculated within a simplified physical model of the surroundings, as well as available data for communication channels at 5.9 $\mathrm{GHz}$, as found in [4].

The key parameters of the OFDM modulator system are the length of the guard interval $T_{G}$, the carrier distance $\Delta f$ and the number of carriers $N$. The frame structure - in particular, its length and the position of pilot symbols - are other parameters which need to be adapted to the distortions caused by the channel.

The reason for these distortions is multipath propagation: the signal reaches its intended destination not only via one, but many paths, each with its own complex attenuation and Doppler shift. The result is a frequency-selective, time-variant channel. Designing OFDM parameters for such channels is a standard problem and has been discussed manifold in literature. To recapitulate, an OFDM system needs to satisfy the following conditions:

1) The guard interval length needs to be larger than the maximum excess delay $\tau_{e}$, i.e. the time difference between arrival of the first and last propagation path.

$$
T_{G}>\tau_{e}
$$

2) The carrier distance $\Delta f$ needs to be smaller than the coherence bandwidth $B_{C}$, i.e. the frequency span over which the channel can be assumed constant. It must also be a lot larger than the Doppler spread $B_{D}$, i.e. the widening of the spectrum as result of the different Doppler shifts, such that the spreading does not destroy orthogonality between carriers.

$$
B_{D} \ll \Delta f<B_{C}
$$

Since OFDM systems are very sensitive to deorthogonalization, a lower channel limit is defined as ten times the Doppler spread.

3) The coherence time $T_{C}$, i.e. the time over which a channel can be assumed approximately constant, must not exceed the time $T_{E}$ between channel estimations, at the very least the time of one OFDM symbol.

$$
T_{E}<T_{C}, \Delta f^{-1} \ll T_{C}
$$

As will become clear in Section IV, most of these requirements have to hold for both the communication channels (between cars) as well as the radar channels (i.e. the scattered signals reflected towards the transmitter). A propagation path which does not fulfill the conditions will lead to selfinterference.

For further analysis of the channel characteristics, a power attenuation threshold $P_{T}$ is introduced. Propagation paths with a power attenuation of more than $P_{T}$ compared to the strongest
TABLE I

RAY TRACING PARAMETERS

\begin{tabular}{r||c|c}
\hline Property & Urban & Autobahn \\
\hline Vehicle velocity & $50 \mathrm{~km} / \mathrm{h}$ & $200 \mathrm{~km} / \mathrm{h}$ \\
\hline Environment & Buildings, parking vehicles & Vegetation \\
\hline Snapshots calculated & 250 & 250 \\
\hline Snapshot interval & $40 \mathrm{~ms}$ & $40 \mathrm{~ms}$ \\
\hline
\end{tabular}

propagation path shall not be considered. Throughout this work, $P_{T}$ shall be fixed at $40 \mathrm{~dB}$, which is a very conservative value - for the communication subsystem, this would require the receiver in the worst case to be able to handle signal to self-interference ratios of at most $40 \mathrm{~dB}^{1}$, which is a more than reasonable specification. In the case of the radar system, $40 \mathrm{~dB}$ implies the furthest detectable target cannot be more than ten times away than the closest one.

\section{A. Worst case estimations}

An upper bound to the channel parameters can be calculated by assuming conservative values. First, assume the distance to the closest detectable radar target is $200 \mathrm{~m}$ and the furthest communication partner is $400 \mathrm{~m}$ away. These values are unlikely to be achieved at $24 \mathrm{GHz}$ using broadband OFDM, but shall be used for an upper bound. In this case, the longest possible propagation time - and thus the maximum necessary guard interval - in both cases is $1.33 \mu \mathrm{s}$.

For the Doppler spread, assume a maximum relative velocity $v_{\text {rel }}=400 \mathrm{~km} / \mathrm{h}$. The Doppler shift for the scattered radar signal is $f_{D}=2 v_{\text {rel }} f_{C} / c_{0}$, where $f_{C}=24 \mathrm{GHz}$ is the signal's centre frequency and $c_{0}$ the speed of light. This results in a Doppler shift of $17.8 \mathrm{kHz}$.

Since the coherence time and the Doppler spread are related inversely, a worst case coherence time estimate is $1 / 17.8 \mathrm{kHz}$ $=0.06 \mathrm{~ms}$.

\section{B. Ray tracing results}

Two ray tracing simulations were evaluated for this work, subsequently called "Urban" and "Autobahn" scenarios. The former simulates an urban environment with a high density of both parking and moving vehicles, whereas the latter is a model of a highway scenario, with vegetation being the main source of scattering. In both cases, one vehicle acts as transmitter and another as receiver. Main specifications of both scenarios are summarized in Table I.

The ray tracer calculates all possible propagation paths at a given interval, resulting in a so-called snapshot. These paths can then be used to calculate the time-variant channel impulse response. In both scenarios, the scattered propagation paths returning to the transmitting vehicle were also calculated to evaluate the data for the radar case.

The following analyses were performed: for every snapshot, all propagation paths attenuated beyond the threshold $P_{T}$ were

\footnotetext{
${ }^{1}$ This assumes the propagation paths attenuated beyond $P_{T}$ do not make up most of the transmitted energy, which is the case in all observations presented here.
} 
TABLE II

CHANNEL CONSTRAINTS ON THE OFDM PARAMETERS

\begin{tabular}{r||c|c}
\hline Property & Urban & Autobahn \\
\hline RMS excess delay & $0.24 \mu \mathrm{s}$ & $0.86 \mu \mathrm{s}$ \\
\hline RMS Doppler spread & $0.30 \mathrm{kHz}$ & $2.72 \mathrm{kHz}$ \\
\hline Coherence bandwidth & $292.97 \mathrm{kHz}$ & $195.31 \mathrm{kHz}$ \\
\hline Coherence time & $0.36 \mathrm{~ms}$ & $0.03 \mathrm{~ms}$ \\
\hline
\end{tabular}

discarded. From the remaining paths, values for the channel characteristics were calculated as follows:

- The excess delay $\tau_{e}$ was calculated by taking the time of arrival difference between the first and the last arriving propagation path. From all excess delays, the root mean square (RMS) value was calculated.

- Doppler shifts of all propagation paths were analyzed to calculate the Doppler spread $B_{D}$, i.e. the difference between the largest and smallest Doppler shift, and used to calculate the RMS Doppler spread.

- The RMS coherence time $T_{C}$ was estimated by the inverse RMS Doppler spread,

$$
T_{C}=\frac{1}{B_{D}} .
$$

- Path delays were quantized to a regular lattice at a given sampling rate $f_{S}$, such that $\tau_{m}=k / f_{S}$ with $k \in \mathbb{N}$. If the channel is assumed time-invariant, the Doppler shifts can be ignored and the channel can be represented by its impulse response $h(k)$.

- By taking the z-transform $H_{z}(z)$ of $h(k)$, the channel frequency response is calculated as $H(f)=H_{z}\left(e^{j 2 \pi f / f_{s}}\right)$. $\rho_{H, H}(f)$ is the normalized autocorrelation coefficient of the channel frequency response.

- The coherence bandwidth was estimated by the value $B_{C}$ where the correlation coefficient of the frequency response $\rho_{H, H}(f)$ drops to $90 \%$, i.e.

$$
\rho_{H, H}\left(B_{C}\right)=0.9 \text {. }
$$

Channel constraints for the OFDM parametrization were then derived as those values for which conditions 1) - 3) were satisfied $95 \%$ of the time ${ }^{2}$. This tolerance level avoids having to account for extreme channel situations which would otherwise cause unnecessarily conservative parametrizations.

Table II shows the resulting values for the channel characteristics, which are less constrictive than the worst case estimates. These will constitute the first set of constraints for the OFDM design.

When comparing these values to the real measurements obtained in [4], one can see these estimations make physical sense: the maximum excess delay and the coherence bandwidth here are less restricting due to the higher attenuation at $24 \mathrm{GHz}$ and Doppler spread and coherence time are worse as cause of the higher Doppler shift.

\footnotetext{
${ }^{2}$ The same method is used in [4], at a tolerance level of $90 \%$.
}

\section{RADIO SYSTEM CONSTRAINTS}

\section{A. Power limitations}

In practical implementations, mobile receivers and transmitters are always limited in their power consumption, effectively limiting the signal-to-noise ratio (SNR) at the receiver. Both radar and communication systems require a minimum SNR to work accurately.

Free space loss shall be used to estimate the received power. In case of communication, the received power $P_{R}$ in a distance of $r$ for a signal at centre frequency $f_{C}$ is

$$
P_{R}=\frac{P_{T} c_{0}^{2}}{(4 \pi)^{2} r^{2} f_{C}^{2}} .
$$

In the radar case, the radar cross section $\sigma$ of the target must be known. Here, the received scattered power ${ }^{3}$ is

$$
P_{R}=\frac{P_{T} c_{0}^{2} \sigma}{(4 \pi)^{3} r^{4} f_{C}^{2}} .
$$

At the receiver, the symbol energy per noise power density in an OFDM system is

$$
\frac{E_{s}}{N_{0}}=\frac{P_{R}}{N \Delta f N_{0}} .
$$

Given minimum values $\left(E_{b} / N_{0}\right)_{\text {min,radar }}$ and $\left(E_{b} / N_{0}\right)_{\text {min,com }}$ for radar and communication reliability, one obtains another constraint for the system design by inserting (2) and (3) into (4):

$$
\begin{gathered}
N \Delta f \leq \min \left(\frac{P_{T} c_{0}^{2}}{\left(E_{b} / N_{0}\right)_{\min , \mathrm{com}} N_{0}(4 \pi)^{2} f_{C}^{2} r_{\text {max }, \mathrm{com}}^{2}},\right. \\
\left.\frac{P_{T} c_{0}^{2} \sigma}{\left(E_{b} / N_{0}\right)_{\text {min,radar }} N_{0}(4 \pi)^{3} f_{C}^{4} r_{\text {max,radar }}^{4}}\right) .
\end{gathered}
$$

As can be seen clearly in the above equation, keeping the bandwidth $N \Delta f$ small is desirable.

\section{B. Hardware limitations}

As mentioned in Section I, the system design shall not be restricted here by external limitations such as price or availability of components. Nevertheless, it is worth describing how the optimization process affects the hardware requirements.

The signal bandwidth $N \Delta f$ is an obvious factor, since for the OFDM demodulation process, the entire signal needs to be converted to a digital signal; thus, keeping bandwidth low will ease hardware requirements.

Peak-to-average power ratio (PAPR) is another OFDM problem which affects the requirements for the transmit amplifier and the dynamic range at the receiver, which is why it is desirable to keep PAPR low. According to [5], PAPR will not exceed a value of $2 \ln N$ with high probability for a large number of carriers; so while reducing $N$ will reduce PAPR, the effect is negligible, and the issue is better addressed somehow else, e.g. by PAPR-reducing coding methods.

\footnotetext{
${ }^{3}$ In both cases, antenna gains are set to $G=1$.
} 


\section{OFDM RADAR}

Let one OFDM frame consist of $N$ sub-carriers and $M$ OFDM-symbols. In complex baseband, the $k$-th sub-carrier is to have the frequency $f_{k}$. Every OFDM symbol consists of $N$ modulation symbols $c_{k, l} \in \mathcal{A}$, with $\mathcal{A} \subset \mathbb{C}$ being a complex modulation alphabet such as QPSK. In OFDM systems, the length of one entire OFDM symbol is

$$
T_{O}=\Delta f^{-1}+T_{G} .
$$

Using these notations, one OFDM frame can be modeled as a matrix $\mathbf{C}_{\mathrm{OFDM}} \in \mathbb{C}^{N \times M}$, which consists of the elements $\left(\mathbf{C}_{\mathrm{OFDM}}\right)_{k, l}=c_{k, l} e^{j 2 \pi l T_{O} f_{k}}$.

In other words, the signal containing one frame consists of $N$ parallel narrow-band signals of length $T_{F}$, or, alternatively, of $M$ sequential wide-band signals of bandwidth $N \Delta f$. The received signal differs from the transmitted signal in two respects: first, it is time-delayed as a cause of the distance $d$ it has to cover and second, it is Doppler-shifted through the relative speed $v_{r}$ of receiver and target. The time delay $\tau$ and the Doppler shift $f_{D}$ are given by the well-known equations

$$
\tau=\frac{2 d}{c_{0}}, f_{D}=\frac{2 f_{c} v_{r}}{c_{0}},
$$

$c_{0}$ and $f_{c}$ being the speed of light and the signal's centre frequency, respectively.

For details of the radar processing, cf. [3]. For the parametrization it is important to note that the measurement resolutions for speed $\Delta v$ and distance $\Delta d$ are given by

$$
\Delta d=\frac{c_{0}}{2 N \Delta f}, \Delta v=\frac{c_{0}}{2 T_{F} f_{c}}
$$

$N \Delta f$ is a measure for the bandwidth of the OFDM signal.

One can tell the radar subsystem performs better if it occupies a high bandwidth, which can be achieved either by increasing the carrier distance or the number of sub-carriers; and if it occupies a longer period of time, which is achieved by adding more OFDM symbols to a frame or increasing the length of one OFDM symbol (and hence decreasing the sub-carrier distance). The $802.11 \mathrm{p}$ standard, which occupies a bandwidth of approximately $10 \mathrm{MHz}$, is clearly not suitable for radar imaging.

\section{A. OFDM radar design margins}

Defining a maximum resolution for speed and range measurements $\Delta d_{\max }$ and $\Delta v_{\max }$ yields a set of inequalities for the system design by inserting these values into (8) and solving for the remaining variables:

$$
\begin{aligned}
& N \Delta f \geq \frac{c_{0}}{2 \Delta d_{\max }} \\
& T_{F} \geq \frac{c_{0}}{2 \Delta v_{\max } f_{c}}
\end{aligned}
$$

The former specifies the signal bandwidth and the latter the frame duration $T_{F}=M\left(1 / \Delta f+T_{G}\right)$.
TABLE III

EXAMPLE SYSTEM SETUP

\begin{tabular}{r|c}
\hline Range accuracy & $\Delta d<1 \mathrm{~m}$ \\
\hline Speed accuracy & $\Delta v<2 \mathrm{~m} / \mathrm{s}$ \\
\hline Communication link error rate & $P_{b}<10^{-3}$ \\
\hline Transmit power & $P_{T}<1 \mathrm{~W}$ \\
\hline Receiver noise power density & $N_{0}=-160 \mathrm{dBm} / \mathrm{Hz}$ \\
\hline Reliable communication distance & $r_{\max , \text { comm }}=100 \mathrm{~m}$ \\
\hline
\end{tabular}

\section{A DESIGN EXAMPLE}

By applying this set of constraints, the OFDM system can be defined. First, we define a set of system reliability parameters such as those in Table III. Applying these particular values, we can use $\Delta d_{\max }=1 m$ with (9) to obtain $N \Delta f \geq 91.5$ MHz. In the case of BPSK and QPSK modulation, the bit error rate before coding for the additive white Gaussian noise (AWGN) case is $P_{b}=0.5 \operatorname{erfc}\left(\sqrt{E_{b} / N_{0}}\right)$, so a minimum $E_{s} / N_{0}$ would be $6.8 \mathrm{~dB}$ for BPSK and $9.8 \mathrm{~dB}$ for QPSK, yielding a maximum bandwidth of $258 \mathrm{MHz}$ and $129 \mathrm{MHz}$, respectively, when applied to (5). Following Section III, the minimum possible bandwidth should be chosen.

Next, the minimum frame length $T_{F}$ is determined using (10) to be $3.1 \mathrm{~ms}$. This has several implications: first, this is a lot more than the channel coherence time, so the equalizer needs to be readjusted several times per frame. Second, at the given bandwidth and frame length, an entire frame consists of several hundred kbit of data. This amount of data has to be transmitted, if necessary by padding the frame with random bits or increasing the channel coding rate.

The frame structure must also be chosen according to the given limitations. A simple solution would be to send two OFDM symbols for synchronization, gain control and equalization and then send one midamble OFDM symbol for re-equalization every $T_{C}$.

Finally, appropriate values for $N$ and $\Delta f$ shall be found. Figure 1 shows the number of carriers for different values of carrier distance at $N \Delta f=91.5 \mathrm{MHz}$. Although the bandwidth is fixed, changing $\Delta f$ affects the symbol rate, as it maximizes the length of one OFDM symbol, thus decreasing the relative length of the guard interval. Of course, by doing so, the length of the synchronization symbols is also increased, which in turn has a negative effect on the data rate.

A good choice for $\Delta f$ is thus the value which maximizes the data rate. For the given frame structure with $n_{S}$ synchronization OFDM symbols, the effective data rate is given by

$$
R=\left(\frac{T_{F}}{\frac{1}{\Delta f}+T_{G}}-n_{S}\right) \frac{N}{T_{F}},
$$

i.e. the number of effective data OFDM symbols divided by the frame length. Figure 1 shows how $N$ and $R$ change depending on $\Delta f$ for a fixed bandwidth of $91.5 \mathrm{MHz} . n_{S}$ was approximated by $n_{S}=T_{F} / T_{C}$. In this particular case, $R$ has a maximum at $\Delta f=76.25 \mathrm{kHz}$.

The final OFDM setup results are summarized in Table IV. 


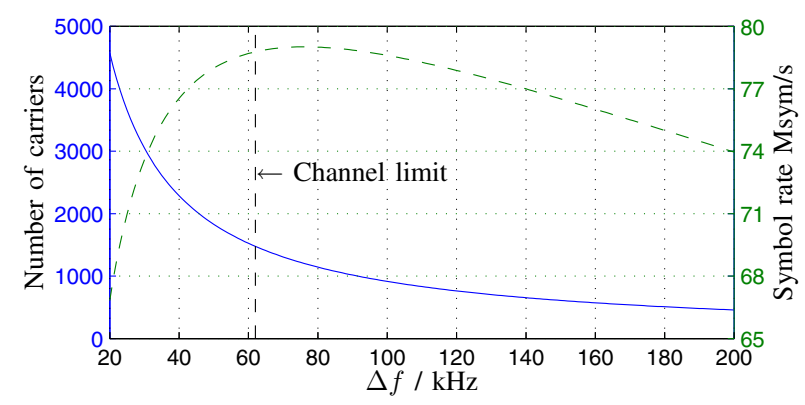

Fig. 1. Number of carriers and symbol rate (dashed line) depending on $\Delta f$.

TABLE IV

EXAMPLE SYSTEM SETUP

\begin{tabular}{r|c}
\hline Carrier distance $\Delta f$ & $76.25 \mathrm{kHz}$ \\
\hline Guard interval length & $1 \mu \mathrm{s}$ \\
\hline Number of carriers $\mathrm{N}$ & 1200 \\
\hline Frame duration & $3.1 \mathrm{~ms}$ \\
\hline Number of OFDM symbols per frame & 220 \\
\hline Number of preamble symbols & 2 \\
\hline Number of data symbols between midamble & 13 \\
\hline
\end{tabular}

\section{A. Simulation results}

In order to verify the communication setup, the channels obtained by the ray tracing simulations were applied to a communication link. Figure 2 shows the attenuation and the bit error rate during the process. 100 frames were transmitted through the channel at each of the 250 channel states. At first, when the vehicles are still far apart, the attenuation is high and the bit error rate is close to 0.5. As soon as the attenuation drops below $100 \mathrm{~dB}$ - the free space attenuation for which the system was designed at the given maximum communication distance - the bit error rate drops to near zero. The residual bit error rate which can be seen above $100 \mathrm{~dB}$ is explained by the fact that the attenuation is calculated by the entire accumulated energy at the receiver, not accounting for fading effects which can increase the bit error rates when the initial OFDM conditions are no longer fulfilled, as is the case around snapshot 150. Furthermore, the employed synchronization method also introduces errors. The effect of the synchronization method was not analyzed in depth for this work.

The necessity for equalization during the frame demodulation can be seen in Figure 3. This graph shows how the bit error rate increases with every OFDM symbol when assuming the channel to be time-invariant. It also suggests that the midamble-every-nth-frame equalizer structure is a possible solution, but not necessarily the most effective one. A better understanding of the different mobile channels at $24 \mathrm{GHz}$ might enable the use of channel correlation properties to enhance the equalization.

\section{CONCLUSION AND FUtURE WORK}

The design process of an OFDM radar and communication system was explained and a possible configuration was pre-

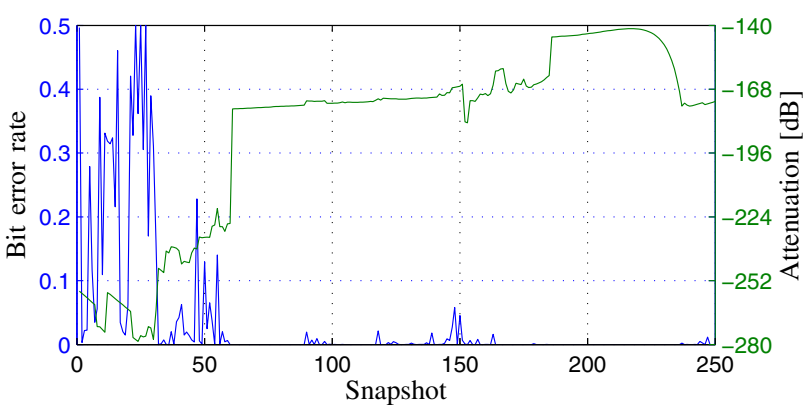

Fig. 2. Bit error rate and attenuation during the simulation

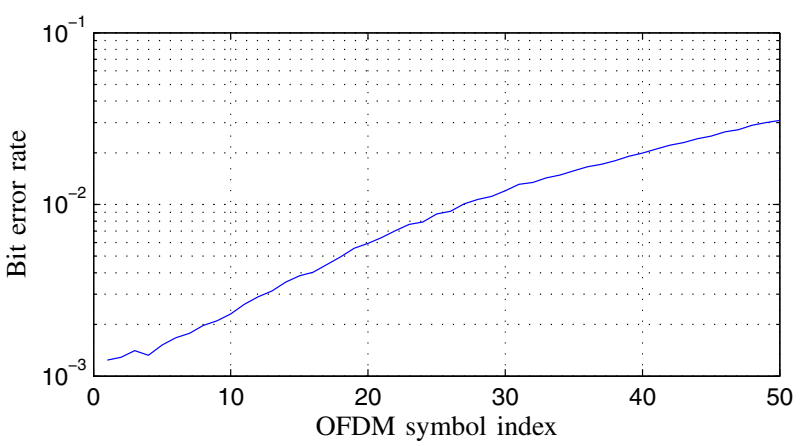

Fig. 3. Bit error rate per OFDM symbol without equalization

sented. Simulations confirm the feasibility of such a system. Future work will include a performance analysis of the radar subsystem and its dependence on $E_{b} / N_{0}$. Furthermore, the frame structure will be analyzed in greater detail, including more efficient pilot symbol structures as well as channel codings. MIMO processing for radar- and communication subsystems and modeling of multi-user scenarios is the focus of current research.

\section{ACKNOWLEDGEMENTS}

The authors gratefully acknowledge that their work is supported within the priority program No. 1163 (TakeOFDM) by the German Research Foundation (DFG).

\section{REFERENCES}

[1] B. Donnet and I. Longstaff, "Combining MIMO Radar with OFDM Communications," Radar Conference, 2006. EuRAD 2006. 3rd European, pp. 37-40, Sept. 2006.

[2] H. Zhang, L. Li, and K. Wu, "24GHz Software-Defined Radar System for Automotive Applications," Wireless Technologies, 2007 European Conference on, pp. 138-141, Oct. 2007.

[3] C. Sturm, E. Pancera, T. Zwick, and W. Wiesbeck, "A Novel Approach to OFDM Radar Processing," IEEE Radar Conference, 2009., May 2009.

[4] L. Cheng, B. Henty, R. Cooper, D. Standi, and T. F. Bai, "A Measurement Study of Time-Scaled 802.11a Waveforms Over The Mobileto-Mobile Vehicular Channel at $5.9 \mathrm{GHz}, "$ Communications Magazine, IEEE, vol. 46, no. 5, pp. 84-91, May 2008.

[5] N. Dinur and D. Wulich, "Peak-to-Average Power Ratio in high-order OFDM," Communications, IEEE Transactions on, vol. 49, no. 6, pp. 1063-1072, Jun 2001. 